\title{
INVESTIGACIÓN/RESEARCH
}

\section{INFLUENCIA DEL USO LAS REDES SOCIALES EN ESTUDIANTES HISPANOHABLANTES EN EL TIEMPO DE OCIO Y DE ESTUDIO}

María del Mar Soria Ibáñez ${ }^{1}$ : Universidad de Málaga. España msoriaibanez@gmail.com

\section{RESUMEN}

El presente trabajo es una radiografía representativa del uso de las redes sociales por parte procedentes de estudios medios y superiores, y la posible influencia de la citada herramienta en su proceso de aprendizaje. Partiendo de que las rutinas diarias de los discentes están condicionadas por su pertenencia a alguna comunidad virtual, ahondamos en las diferencias y similitudes que se establecen entre los diferentes estudiantes hispanohablantes en cuanto al uso de las redes sociales. La metodología elegida ha sido la encuesta y el estudio es un análisis reflexivo y representativo que refleja la situación actual de una parte de la comunidad discente de cada uno de los países seleccionados, sin que conduzca a conclusiones categóricas, pero sí orientativas.

PALABRAS CLAVE: Estudiantes - Redes sociales - Socialización - Web 2.0 Educación.

\footnotetext{
${ }^{1}$ Autor correspondiente:

María del Mar Soria Ibáñez: Periodista y Doctora en Comunicación por la Universidad de Málaga. España.
}

Correo: msoriaibanez@gmail.com 


\section{INFLUENCE OF SOCIAL MEDIA USE IN STUDENTS SPEAKING IN THE LEISURE AND STUDY TIME}

\section{ABSTRACT}

This is an x-ray of the use of social networks for part of superior and media studies, and the possible influence of tool in their learning process. Based on the daily routines of the learners are conditioned by their membership to a virtual community, we delve into the differences and similarities between the different Spanish-speaking students in the use of social networks. The chosen methodology has been the survey and the study is reflective and representative analysis that reflects the current situation of a part of the sutdents of each of the selected countries, unless it leads to categorical conclusions, but indicative.

KEYWORDS: Students - Social networking - Socialization - Web 2.0 - Education

\section{INTRODUCCIÓN}

Desde que en los años 60 surgieran las primeras conexiones de Internet hasta la actualidad, hemos asistido a numerosos avances protagonizados por la llegada del primer navegador, a mediados de los 90, junto al inexorablemente unido nacimiento de la Web 1.0, o, lo que es lo mismo, aquélla que proporcionaba información y servicios al usuario de manera unidireccional.

Sin embargo, la intrépida aparición de la Web 2.0, cuya consolidación sitúan los expertos en el año 2003, demostró a organizaciones y públicos que el usuario era el protagonista, ya que, mediante esta nueva versión de la Red, el público interactúa de manera bidireccional.

Si extrapolamos todo ello a la educación, podemos hablar de múltiples y diferentes herramientas de aplicación académica, tales como el blogo la «webquest».

El trabajo colaborativo a través de Internet también está protagonizando el día a día de todas las aulas. Tal es así, que numerosos estudios avalan las potencialidades de aquéllas aplicaciones que impulsan el trabajo en equipo en Internet (CamarinhaMatos, 2009; Langer\& Georgea, 2003).

Por otro lado, las redes sociales se han convertido en herramienta indispensable para jóvenes estudiantes cuyas rutinas diarias están siempre relacionadas con el entorno social que les rodea.

Estudios como el desarrollado por Parra Castrillón (2010) ya dan cuenta de la importancia de las redes sociales para estudiantes universitarios, y de la influencia de éstas en la vida cotidiana y académica de los discentes. Así, existe una alta frecuencia de empleo de redes sociales, fundamentalmente de Facebook (el $97 \%$ de los 
estudiantes encuestados la utiliza), y no siempre para fines académicos (Parra Castrillón, 2010: 200).

En términos globales, el uso de las redes sociales está muy extendido a nivel mundial. Si atendemos al informe sobre «La Sociedad de la Información en España 2010», podemos hablar de un total de 945 millones de usuarios de redes sociales en el mundo. El país que aglutina un mayor número de adeptos es EE.UU., seguido de China, Alemania, Rusia, Brasil, Reino Unido, India, Francia, Japón y Corea del Sur.

De una manera más concreta, el estudio sobre redes sociales realizado por la empresa The Cocktail Analysis (2010) da cuenta de que la razón fundamental para hacer uso de estas herramientas es la de mantener el contacto con amigos y conocidos, puesto que un $61 \%$ de los españoles encuestados opina de este modo. En segundo lugar estarían los que usan las redes sociales por entretenimiento $(51 \%)$ o por poder localizar a viejos amigos y conocidos (36\%). Dicho esto, y a tenor de lo representado por el siguiente gráfico, de lo que no cabe duda es que las comunidades virtuales generadas en Tuenti o en Facebook tienen como fin último realizar un uso para fines académicos.

Gráfico 1. Motivo de uso de las redes sociales en España The Cocktail Analysis (2010)

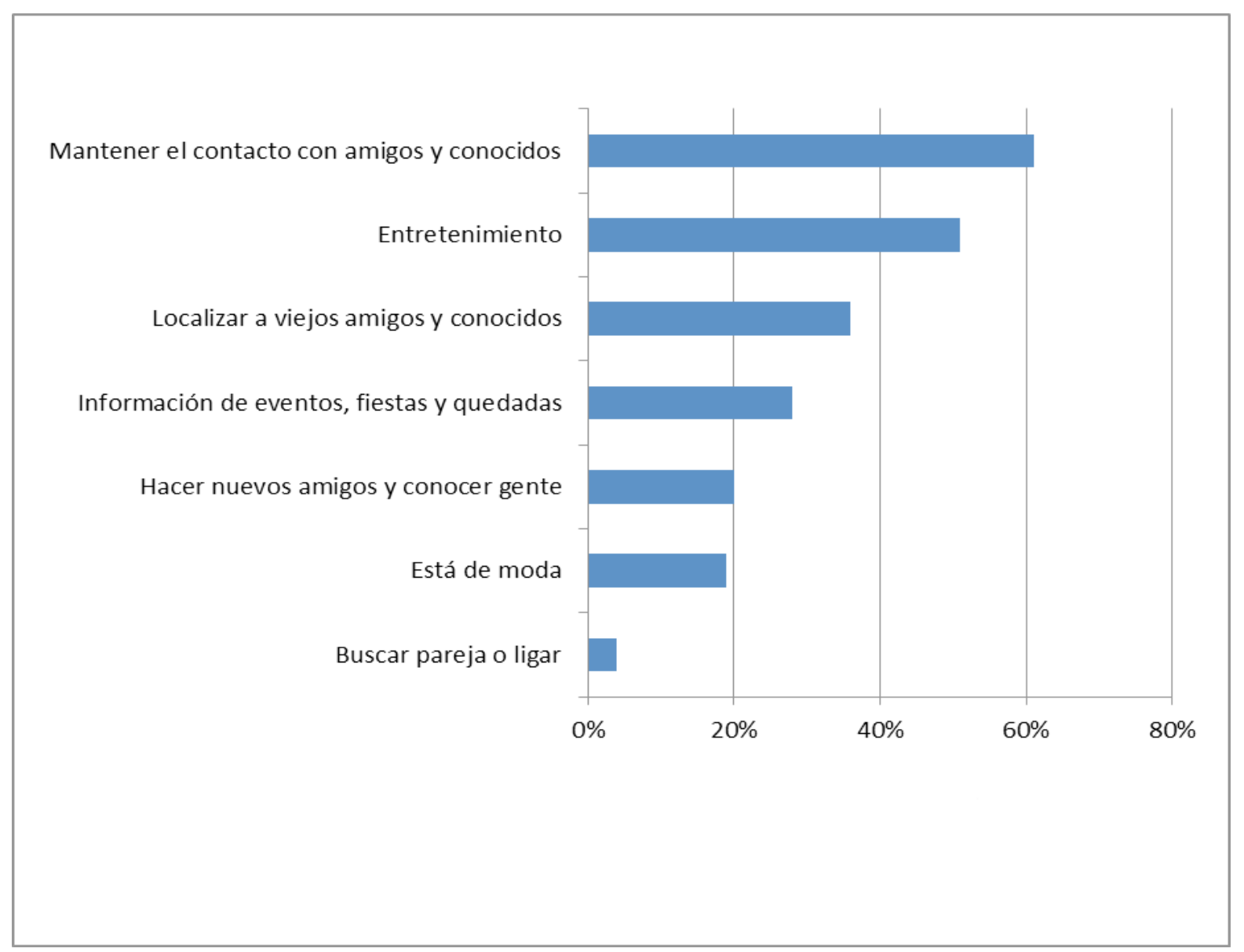




\subsection{Justificación y objetivos de la investigación}

El uso de las redes sociales, muy extendido en todos los países; tienen un fin socializador muy potente, y se han convertido en la herramienta prioritaria para mantener el contacto con las personas ya conocidas o ampliar el círculo social. Así, de una forma u otra, los jóvenes pasan la mayor parte de su tiempo conectados a Internet.

Según Castells (2001: 15), "las redes son formas muy antiguas de la actividad humana, pero actualmente dichas redes han cobrado nueva vida, al convertirse en redes de información impulsadas por Internet", por lo que debemos detenernos en este análisis para poder seguir mirando hacia el futuro bajo una praxis adaptada a las nuevas necesidades.

Y esas nuevas necesidades pasan ya por la competencia digital. Concebida como una habilidad que ha de adquirir el estudiante en su proceso de aprendizaje, y que permite buscar, obtener, procesar y comunicar la información y transformarla en conocimiento a través de las Nuevas Tecnologías de la Comunicación y de la Información.

Es en el contexto de esa competencia digital donde las redes sociales cobran sentido. Pero la realidad es que los centros educativos aún no se han adaptado a las nuevas tendencias de una sociedad digitalizada, y es por ello que un cambio metodológico no es sólo necesario sino que es el único modo de que los estudiantes aprendan mediante su realidad cotidiana:

"Las tendencias virtuales aportadas desde estos nuevos medios, ayudarán, sin duda, a dar significado y a construir una cosmovisión del mundo diferente donde la comunicación, las relaciones mediadas mediante el ordenador y la información definan a la persona. Esta pretensión choca con la realidad que se vive a diario en los centros educativos" (Aznar y Soto, 2010: 85)

Sin embargo, pese a lo anterior, diversos estudios ponen de manifiesto las potencialidades que brinda Internet en el aprendizaje del alumnado, independientemente de las herramientas de las que se haga uso. Es el caso, por ejemplo, de las aportaciones de Weert (2005) o de Barron, A.; Gary W.O (1997), quienes ya vaticinaron, incluso a finales de la década de los noventa, que Internet podría convertirse en una herramienta elemental para el diseño del curriculum en educación.

Así, nos proponemos reflejar la situación actual en torno al uso (académico o no) de las redes sociales en los principales países de hispanohablantes. Conocer el nivel de penetración de las comunidades virtuales en estos estudiantes, y el establecimiento de diferencias y similitudes entre todos ellos, nos llevará a determinar si podría resultar útil abordar parte de la enseñanza desde las redes sociales. Debemos añadir que se trata de una muestra representativa de un grupo de discentes y que esta investigación no refleja la totalidad de la población de los países objeto de estudio, dado que ello 
requeriría un estudio más exhaustivo y complejo, de mayor duración, y éste no es el caso.

Dicho lo anterior, nos proponemos los siguientes objetivos:

1. Ahondar en el papel socializador de la red social de la que hace uso el estudiante, con el fin de determinar el grado de penetración de Internet en la vida social del alumnado.

2. Conocer si el lugar de procedencia y la edad del estudiante condiciona el uso de una u otra red social, de modo que este criterio se tenga en cuenta desde el punto de vista docente para abordar la práctica de un modo u otro.

3. Determinar el tipo de redes sociales más utilizadas y ahondar en el tiempo medio dedicado a esta tarea por estudiantes de estudios medios y superiores, de manera que esto nos lleve a saber si una metodología educativa que haga uso de esta herramienta sería rentable, dependiendo del tiempo que el discente dedique al uso de Internet.

4. Concluir si el uso de las redes sociales como soporte educativo sería factible, partiendo de la relación actual que se establece entre estudiantes e Internet y la posible vinculación con la vida académica.

Cabe matizar que los fines de esta investigación se basan en una muestra no representativa, pero sí lo suficientemente característica para poder ofrecer una visión global y orientativa sobre la influencia de las redes sociales en el trabajo de los estudiantes hispanohablantes.

\section{METODOLOGÍA}

La metodología empleada para desarrollar nuestro estudio y alcanzar los objetivos propuestos ha sido la encuesta. Se trata de una técnica de investigación cuantitativa que puede definirse como "una interrogación particular respecto de una situación que comprende a ciertos individuos con el objetivo de establecer una generalización" (Ghiglione \& Matalon, 1989: 13).En el caso concreto de la presente investigación, hemos hecho de una encuesta formada únicamente por preguntas cerradas, es decir, aquéllas cuyo modelo responde a un "cuestionario estandarizado, unas preguntas redactadas de antemano, un orden preestablecido y unas respuestas de elección limitada" (Visauta (1989: 243).

Dicho lo anterior, las 20 preguntas lanzadas a la población objeto de estudio se han estructurado atendiendo a los siguientes criterios:

1. Perfil de los estudiantes: lugar de origen, tramo de edad, estudios que se está cursando (estudios medios/estudios universitarios) o sexo.

2. Uso de Internet: tiempo de conexión diaria o herramientas más utilizadas.

3. Uso de redes sociales: motivaciones, tiempo dedicado, formas de uso, socialización o empleo didáctico de las mismas. 


\subsection{Población}

Aunque la encuesta estaba dirigida, inicialmente, a 1.000 estudiantes en total (500 de estudios medios y 500 de estudios superiores), respondieron finalmente 900 , de los cuales 430 pertenecían a estudios de secundaria y 470 a estudios universitarios. Por tanto, insistimos, la pequeña muestra no es representativa del total de la población, por lo que las conclusiones de esta investigación son orientativas.

Además, la encuesta estaba dirigida a estudiantes de nacionalidad argentina, chilena, mejicana y española, de manera que, aunque en un principio se estimó una población total de 1.000 personas, las preguntas fueron remitidas a cuatro grupos distintos divididos por nacionalidad.

Hablamos, por tanto, de muestra por conglomerados que aglutina a discentes de estudios medios (de entre 12 y 18 años) y de estudios universitarios (más de 18 años), de clase media y procedentes de Argentina, Méjico, España y Chile.

La encuesta fue realizada a la población seleccionada entre mayo y julio de 2012.

Para el envío de las preguntas a los estudiantes de distintos niveles y nacionalidades, se contó con la ayuda de diversos organismos que colaboraron en su difusión.

Recordamos, además, que llegamos a una media de 225 estudiantes por nacionalidad, si bien es cierto que el nivel de respuesta fue ligeramente desigual, aunque en la práctica poco variable; por tanto, y dada la tasa de respuesta, en ningún caso podemos hablar de un estudio representativo de la población abordada, pero sí orientativo.

\section{ANÁLISIS Y DISCUSIÓN}

\subsection{Los perfiles de los estudiantes}

La mayor tasa de respuesta se ha dado entre el alumnado de nacionalidad española, si bien se debe señalar que la encuesta se repartió por igual número entre los distintos estudiantes seleccionados. De este modo, nos hemos encontrado con que un $55 \%$ de los estudiantes que han respondido son de nacionalidad española, mientras q un $25 \%$ proceden de Argentina, un $12 \%$ de Chile y un $8 \%$ de Méjico. 
Gráfico 2. Porcentaje de respuestas por nacionalidad

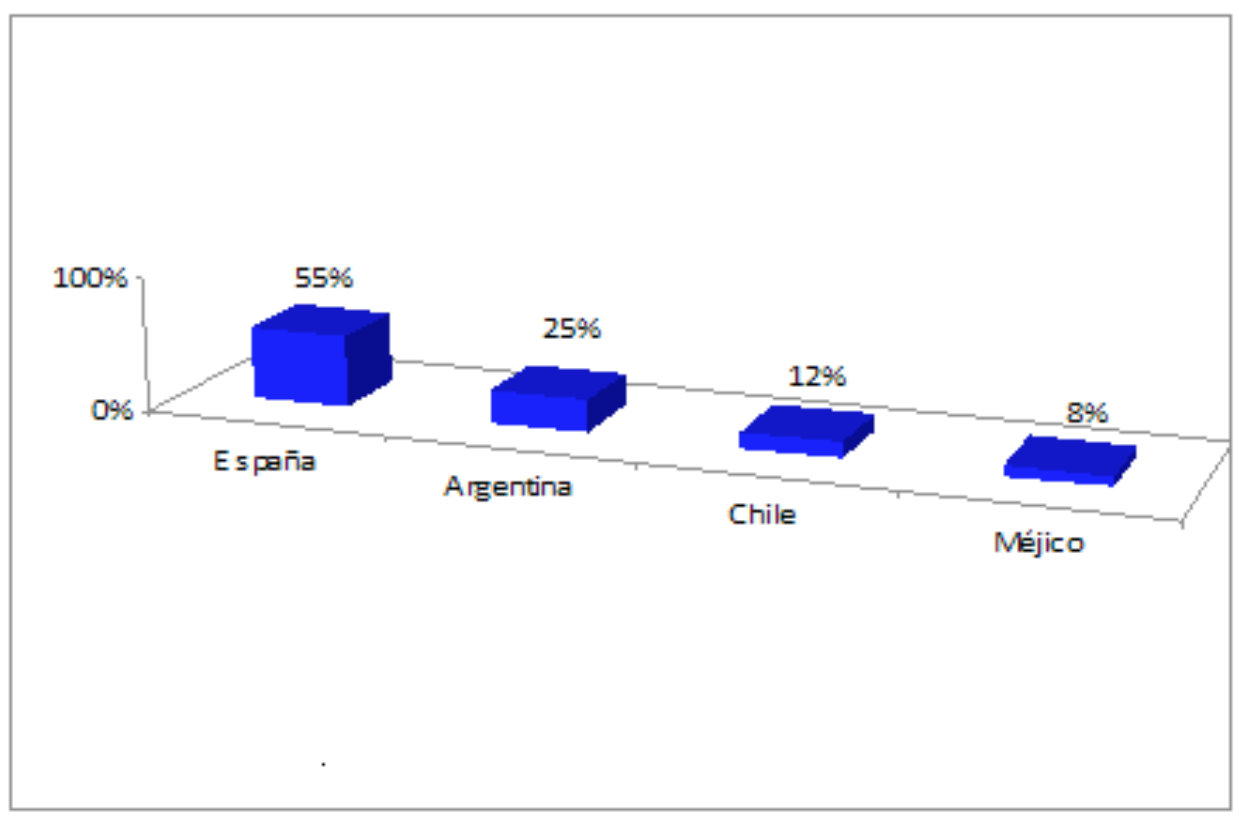

Siguiendo con las tasas de respuesta, también debemos señalar que el grupo de estudiantes que más ha destacado ha sido el que tiene una edad por encima de los 18 años, o, lo que es lo mismo, aquéllos que ya estudian en la Universidad, puesto que representan el $57 \%$ de las personas cuestionadas. Después le seguiría el colectivo procedente de estudios medios que tiene una edad comprendida entre los 16 y 18 años, pues representa un $20 \%$ de la tasa de respuesta, mientras que el último grupo siguiendo este orden sería el de aquellos discentes que tienen entre 12 y 15 años (un $23 \%$ de estudiantes tienen este tramo de edad).

Es decir, el alumnado que ha alcanzado cierta madurez académica y que cursa estudios universitarios manifiesta un mayor interés por el tema que hemos estudiado $y$, por tanto, sus respuestas se tienen en cuenta en esta proporción, puesto que también representan el mayor volumen de resultados.

En la línea anterior, también debemos tener presente, para poder contextualizar mejor los resultados de esta investigación, que un $62 \%$ de las respuestas proceden del género femenino, mientras que un $38 \%$ se desprende del colectivo masculino.

\subsection{El uso de las redes sociales como elemento socializador}

En primer lugar, y antes de centrarnos en el empleo de las redes sociales por parte del alumnado cuestionado, tenemos que subrayar que el $99 \%$ del mismo asegura tener Internet en casa. Hablamos, por tanto, de que solamente una persona, de las 100 que ofrecieron su respuesta, no tiene acceso a Internet desde el hogar. 
Además, el tiempo que se dedica a Internet diariamente también es considerable, pues hablamos de que un $60 \%$ dedica entre dos y cinco horas, independientemente de cuál sea su nacionalidad.

Ahora bien, al preguntarle a los diferentes grupos de estudiantes sobre la herramienta $w e b$ de la que hacen un mayor uso para comunicarse con su entorno, el $62 \%$ coincide en que el soporte óptimo son las redes sociales. Tan sólo un $13 \%$ utiliza los chats para estos fines, mientras que un $25 \%$ prefiere mantener el correo electrónico para esta función

Gráfico 3. Herramientas para comunicarse con el entorno

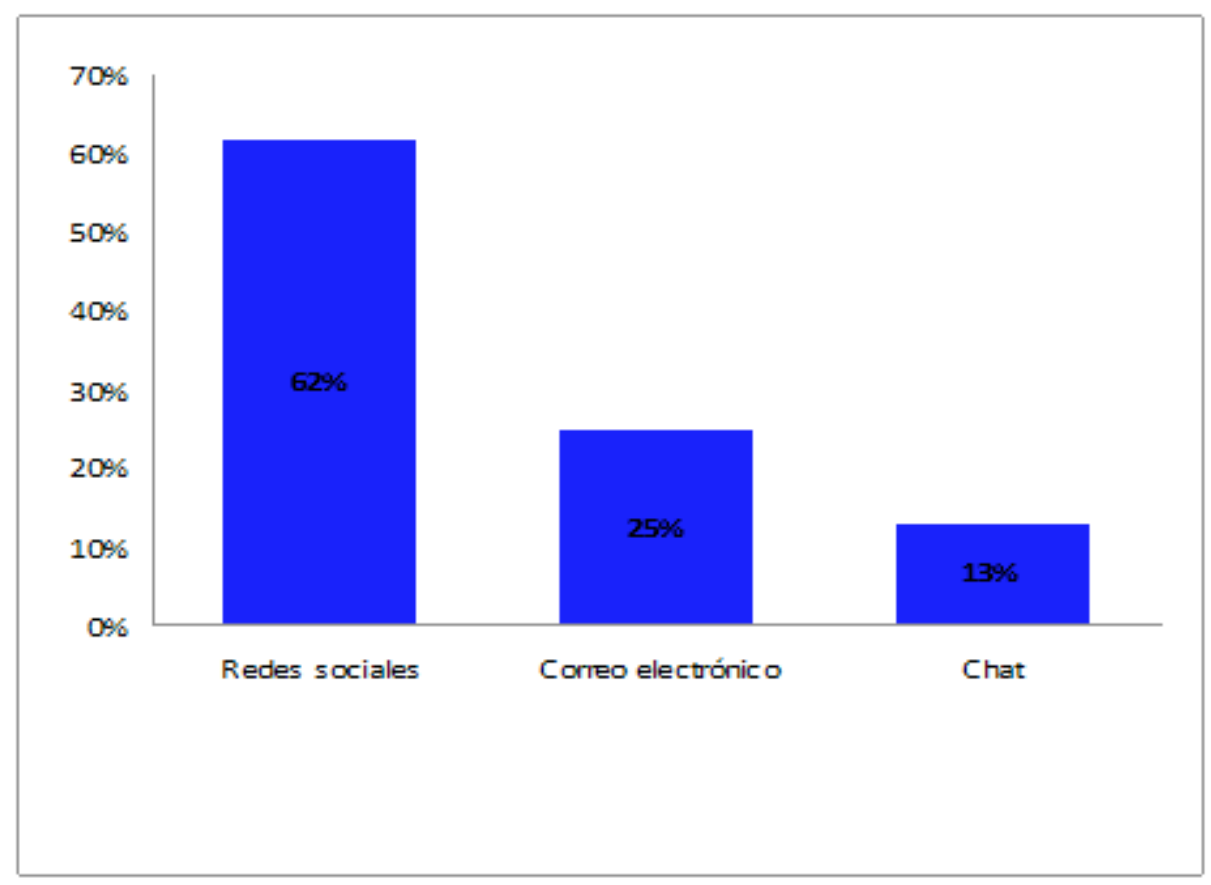

Por otro lado, y en lo que respecta al uso de una red social y otra, hay una clara diferente dependiendo del tramo de edad y nacionalidad. El sexo, en este caso, no es un condicionante para la elección de una u otra comunidad virtual.

Así pues, tal y como nos muestra el siguiente gráfico, la mayor parte de los estudiantes cuestionados pasan entre dos y cinco horas conectados a las redes sociales. Por países, Méjico destaca en el tiempo de conexión, pues un la mayor parte del alumnado del país $(60 \%)$ ha manifestado esta respuesta. Después le seguiría Chile, ya que un $36 \%$ de los discentes, ya sea de estudios medios o superiores, pasa menos de una hora conectado a alguna comunidad virtual.

La media de tiempo de conexión se sitúa, por lo tanto, entre las dos y cinco horas. Estimamos que durante estas horas el estudiante está realizando también otras tareas que simultánea con el contacto permanente con su entorno. 
Gráfico 4.Tiempo que pasan los estudiantes en las redes sociales por nacionalidad

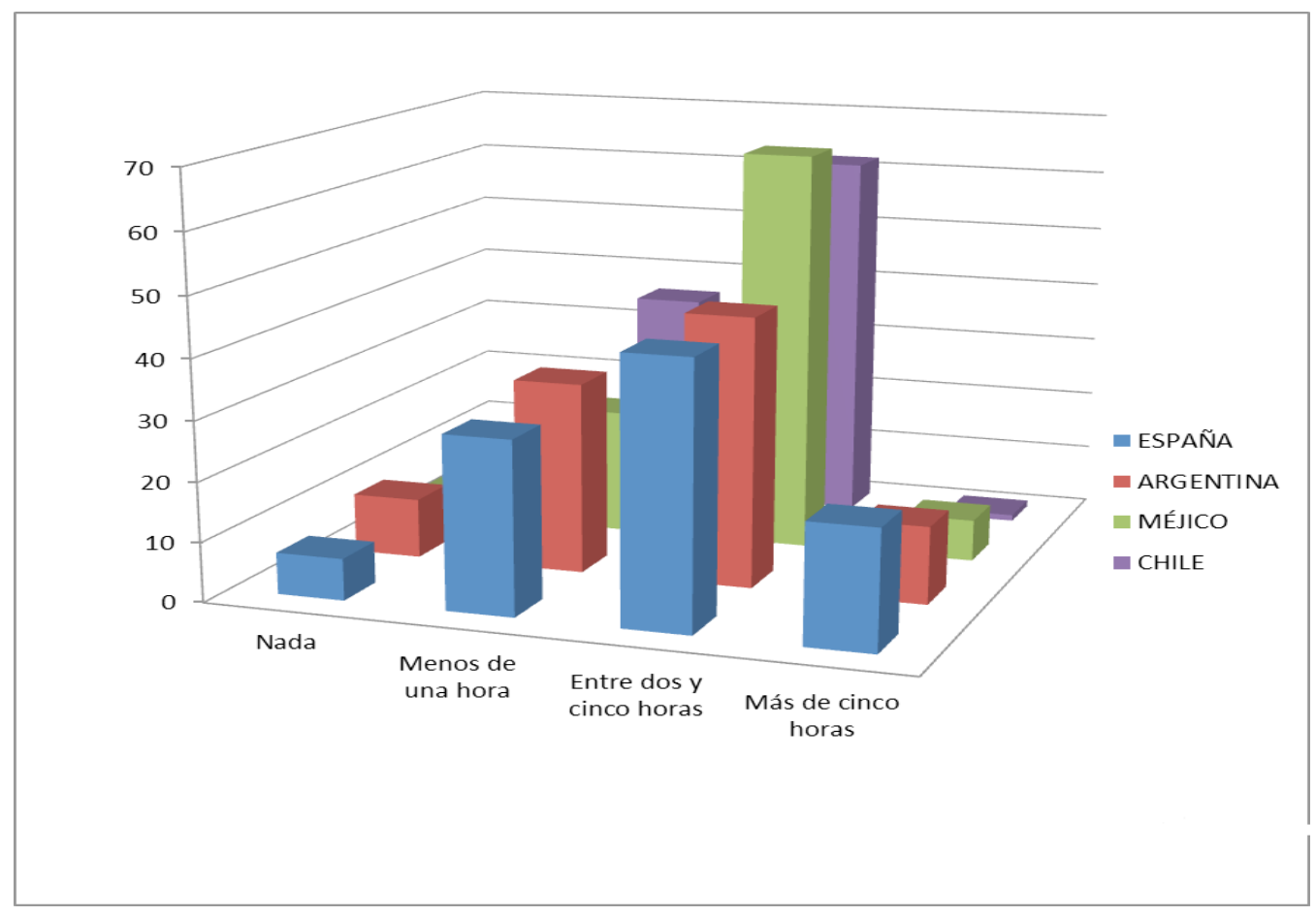

Por otro lado, también encontramos diferencias entre el uso de una u otra red social, teniendo en cuenta que hay una clara prevalencia de Facebook, Tuenti (en el caso de España) y Twitter $^{1}$.

De este modo, la red social Facebook es la más utilizada entre el alumnado, aunque adquiere un especial protagonismo entre los estudiantes de Argentina y de Méjico, de manera que un $78 \%$ de los primeros, y un $80 \%$ de los segundos manifiesta estar integrado dentro de la comunidad virtual. Por su parte, Chile y España se igualan en el empleo de esta red social, ya que el $40 \%$ de los discentes encuestados de ambos países confiesa tener un perfil en Facebook. Todo esto sucede cuando hablamos de una población mayor de 18 años.

Tuenti, por su parte, adquiere una mayor presencia entre el alumnado español, dado que un $30 \%$ del mismo asegura emplear esta herramienta de comunicación social.

Igualmente, resulta interesante que muchos de los estudiantes hayan manifestado pertenecer a varias redes sociales de manera simultánea. Es el caso de Chile, donde el $30 \%$ asegura estar integrado en varias comunidades virtuales. Destacamos, además, que el alumnado chileno es también el que realiza un mayor uso de Twitter $(30 \%)$, una red social que goza de poca popularidad entre las demás nacionalidades encuestadas.

El siguiente gráfico da muestra de todo lo descrito: 
Gráfico 5. Redes sociales usadas por estudiantes de más de 18 años por nacionalidad

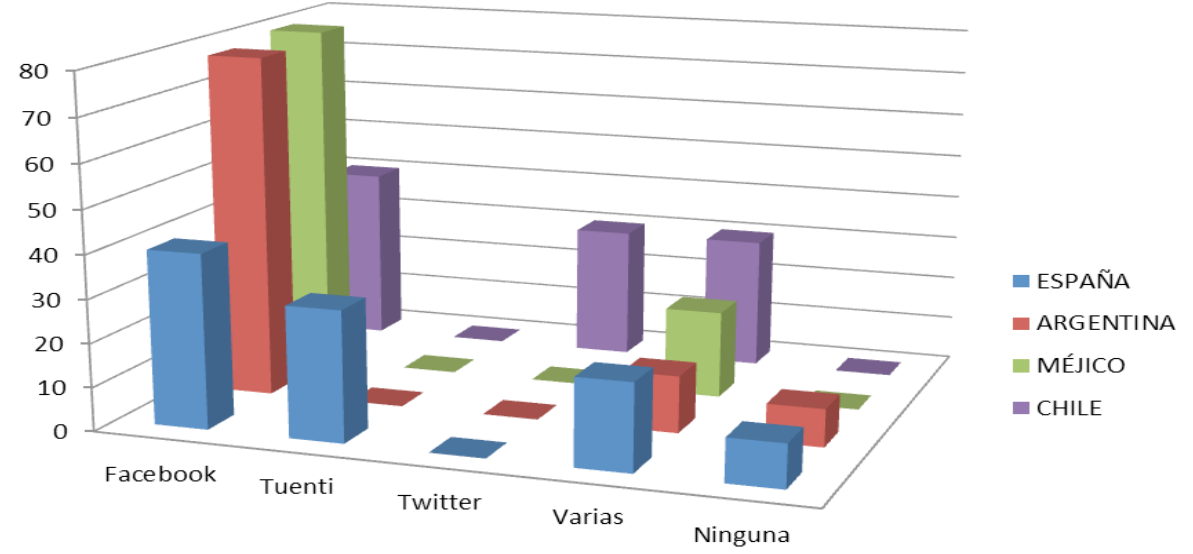

Ahora bien, los datos referidos anteriormente no varían mucho en otros tramos de edades, tal y como se puede observar en los dos gráficos siguientes. De hecho, se puede apreciar que los estudiantes de nacionalidad española de entre 12 y 18 años realizan una clara apuesta por el uso de la red social Tuenti, algo que no ocurría con los discentes mayores de edad y que estudian en la Universidad.

Igualmente, sigue prevaleciendo el uso de Facebook entre el alumnado de Chile y de Méjico en el tramo de edad comprendido entre los 12 y los 18 años, pero no se registran datos de la población argentina en estos períodos. En ambos casos se percibe también que existe entre un $5 \%$ y $10 \%$ de estudiantes españoles que utilizan varias redes sociales a la vez, del mismo modo que en el caso del grupo de españoles que tienen entre 12 y 15 años hay un $12 \%$ que asegura no utilizar ninguna red social.

Gráfico 6. Redes sociales usadas por estudiantes en el tramo de entre 12 y 15 años por nacionalidad

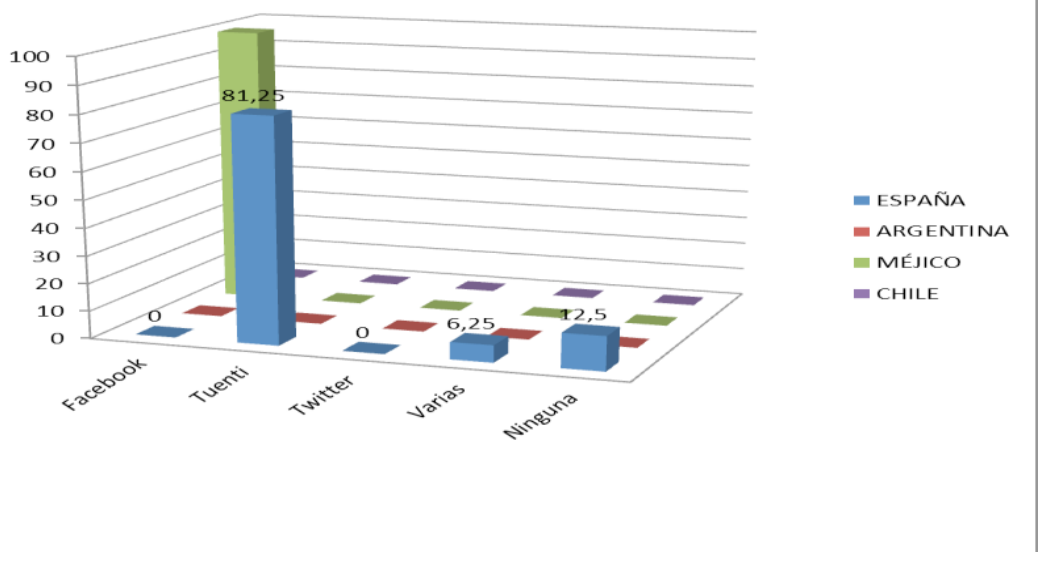


Gráfico 6.Redes sociales usadas por estudiantes en el tramo de entre 16 y 18 años por nacionalidad

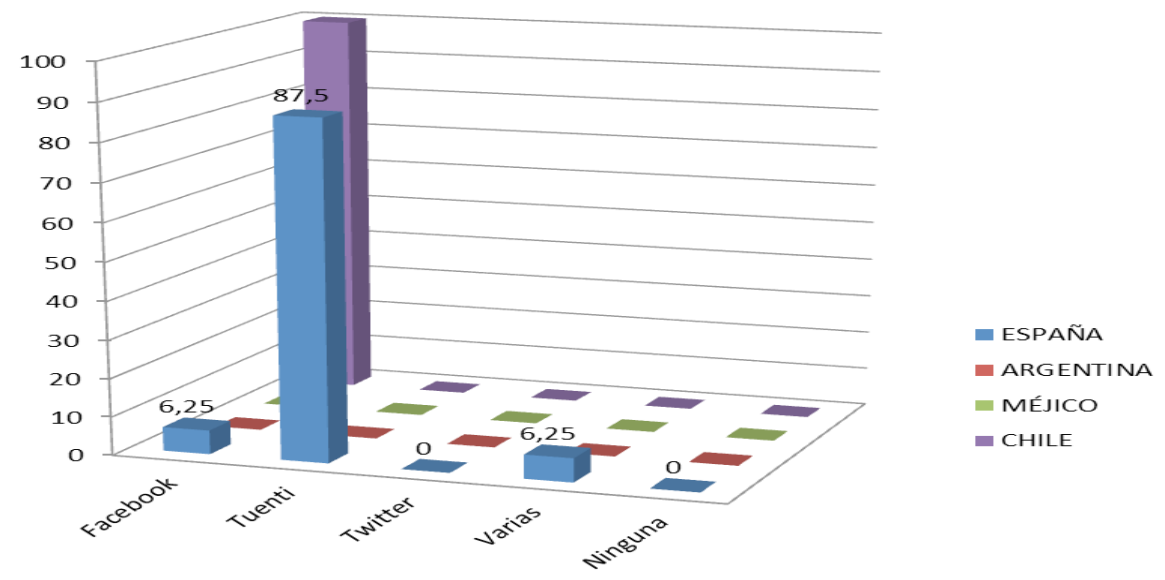

Por otro lado, y siguiendo con la relación que se establece entre el alumnado encuestado y las redes sociales, nos detenemos en el grado de confianza que depositan en esta herramienta. Así, y tal y como se puede apreciar en el gráfico, Tuenti goza de una mayor popularidad, puesto que el $45 \%$ de los estudiantes confían más en esta red social que en cualquier otra. Ello resulta lógico porque, tal y como se ha demostrado entre los gráficos anteriores, el número de adeptos de Tuenti es casi igual al de Facebook, teniendo en cuenta que hablamos de una red social española y cuyos usuarios suelen ser de esta nacionalidad

Gráfico 7. Redes sociales usadas por estudiantes en el tramo de entre 16 y 18 años por nacionalidad
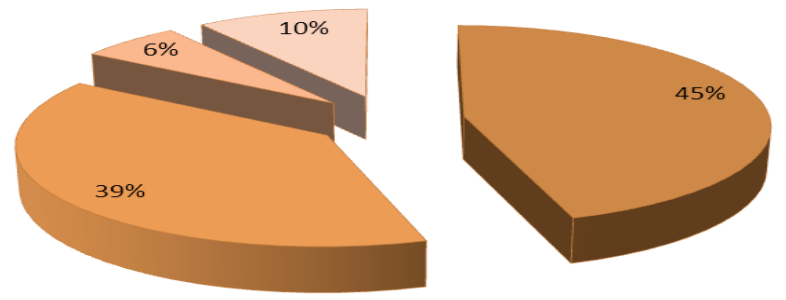

- Tuenti

$\square$ Facebook

$\square$ Twitter

$\square$ Ninguna 
Al hilo de lo anterior, también hemos comprobado que tan sólo el $25 \%$ cree que las redes sociales garantizan la privacidad del usuario, mientras que el $75 \%$ indica que no cree que se salvaguarde su intimidad. Pese a que la mayor parte de los estudiantes manifiesta que el pertenecer a una comunidad virtual puede poner en peligro su intimidad, lo cierto es que, como ya se ha comprobado, la práctica totalidad de las personas cuestionadas se integra en alguna red social, pese a que en muchos casos no se confía en esta herramienta.

Por otro lado, es normal realizar comentarios en el perfil de otros usuarios. El $67 \%$ de los discentes asegura que suele opinar sobre sus amigos/-as más cercanos y comunicarse con ellos mediante comentarios públicos. Tan sólo el $33 \%$ manifiesta no realizar comentarios en el perfil de los contactos con los que está conectado en las redes sociales.

Aunque, como hemos comprobado, las redes sociales no gozan de la total confianza de los jóvenes, la mayor parte de los mismos realiza un uso continuado y permanente de Tuenti o Facebook. Así, al preguntarles sobre el motivo de uso de las comunidades virtuales, la respuesta más repetida (91\%) es la de estar en contacto con personas del entorno. Además, y dado que se les daba la posibilidad de multirespuesta, muchos de los estudiantes también coincidieron en afirmar que emplean las redes sociales para generar conocimiento, si bien este argumento se ha manifestado en un $39 \%$ de los casos.

Gráfico 8. Usos de las redes sociales

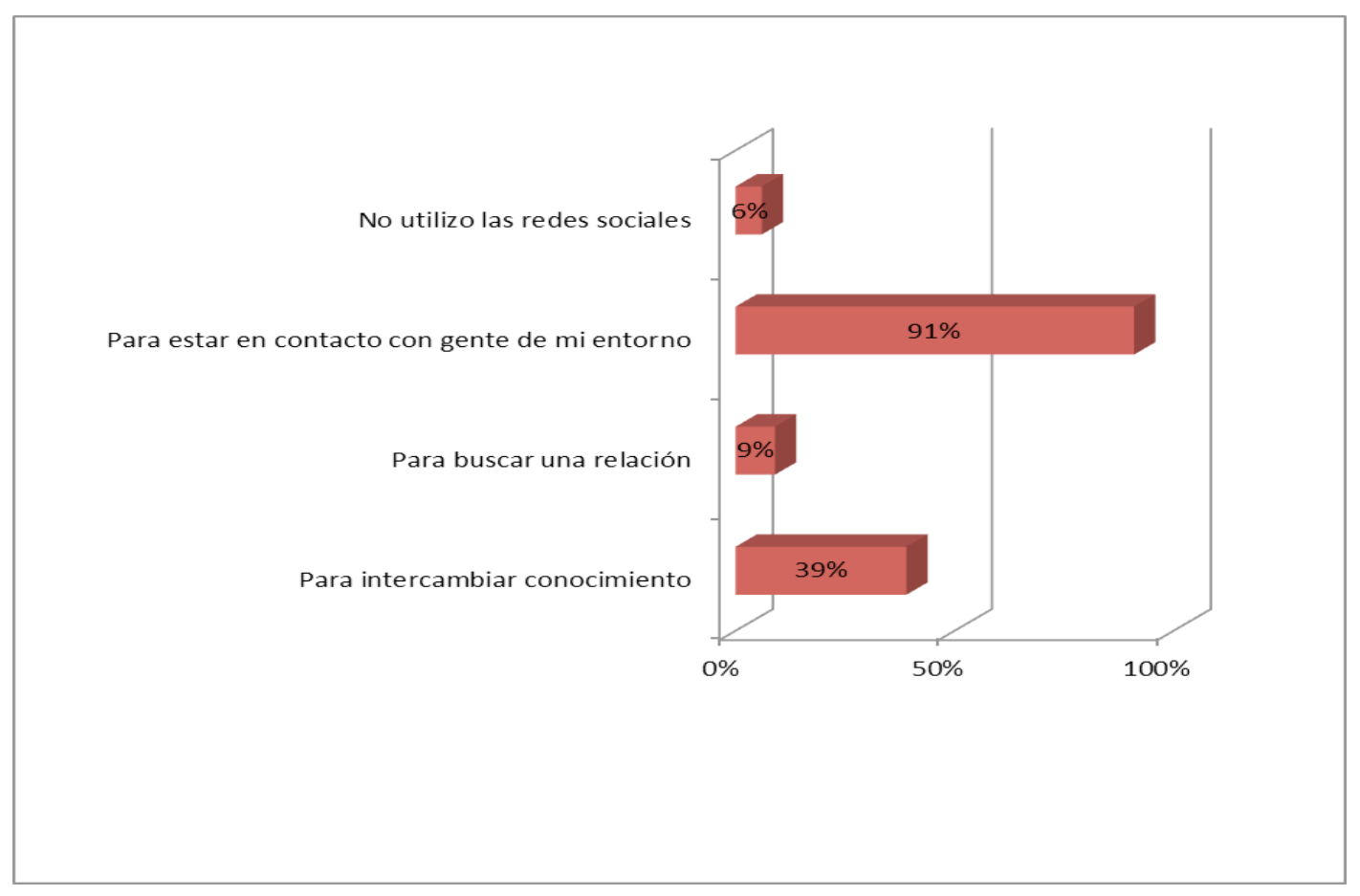

Del mismo modo, las opiniones de los jóvenes a cerca del uso de las redes sociales se orientan hacia el empleo socializador de las mismas. Así, el 76\% de los mismos prefiere estar en contacto real con las personas de su entorno que comunicarse con ellas mediante las comunidades virtuales. Del mismo modo, un $33 \%$ afirma que 
gracias a esta herramienta ha podido aumentar el número de amistades ${ }^{2}$.Queda claro, por tanto, que las redes sociales se convierten en un elemento dinamizador de relaciones sociales.

Finalmente y para terminar con el apartado referido a las redes sociales como elemento de socialización entre los estudiantes hispanohablantes, queda destacar que hay una gran parte de discentes de edades comprendidas entre los 12 y 18 años cuyos contactos en las comunidades virtuales son los mismos que en la vida real, pero también algunas personas que no se conocen directamente. De hecho, el $51 \%$ de los discentes cuestionados así lo han asegurado. Sin embargo, también existe un 47\% que indica que sólo mantiene contacto con personas con las que se relaciona normalmente en su entorno, mientras que un escaso $1 \%$ manifiesta que solamente se comunica con gente desconocida.

\section{3 ¿Uso académico de las redes sociales?}

Teniendo en cuenta que existe una fuerte tendencia a emplear las redes sociales bajo una perspectiva socializadora, hemos de citar también su uso académico, aunque éste no alcance tanta relevancia.

Sin embargo, consideramos relevante arrojar algunos datos a este respecto, puesto que es fundamental para conocer el aprovechamiento de estas comunidades virtuales como herramienta educativa.

Así, en primer lugar, hemos detectado que el $74 \%$ de los estudiantes se comunica a través de las redes sociales para solventar dudas sobre sus estudios, mientras que tan sólo un $26 \%$ jamás realiza este uso.

Por otro lado, podemos apreciar ligeras diferencias entre estudiantes hispanohablantes si nos referimos a una posible influencia negativa del uso de las comunidades virtuales en el rendimiento académico. Así, se destaca el $63 \%$, de los discentes españoles, el $51 \%$ del alumnado argentino, el $40 \%$ del mejicano y el $72 \%$ del chileno aseguran que, en efecto, su tiempo de conexión a las redes sociales afecta negativamente a sus estudios. Sin embargo, y como dato más positivo, hemos comprobado que los estudiantes mejicanos son los que menos se dejan influenciar por el uso desmedido de las redes sociales, puesto que un $45 \%$ de los mismos manifiesta que el tiempo de conexión no influye en su rendimiento académico. 
Gráfico 9. Usos de las redes sociales

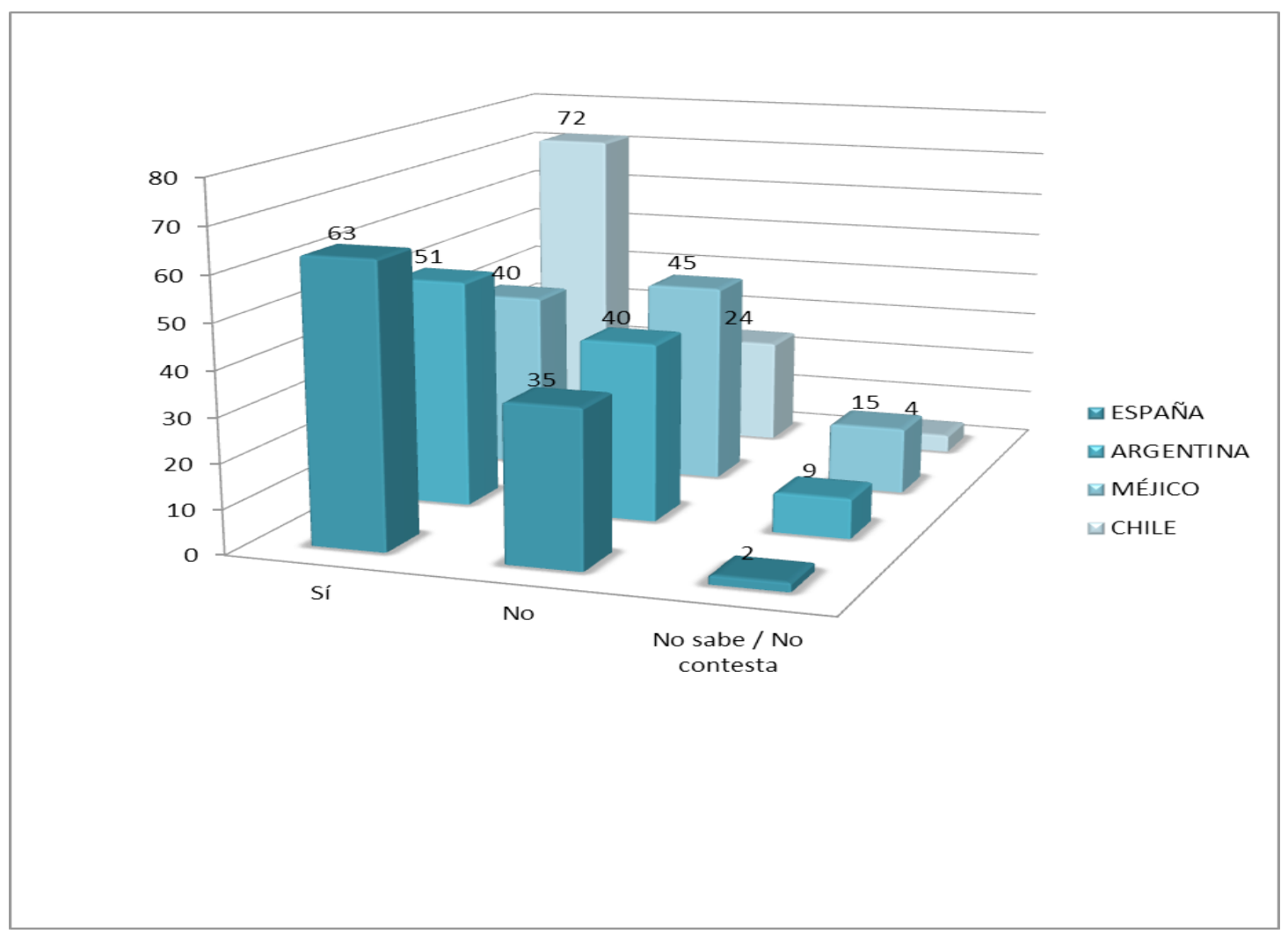

Como vemos, las diferencias en el uso de las redes sociales como herramienta educativa son poco palpables entre los distintos estudiantes entrevistados. De este modo, hemos podido comprobar que el $23 \%$ de los discentes utiliza las comunidades virtuales para completar sus actividades de clase, mientras que el $30 \%$ manifiesta no hacer este uso nunca. El $47 \%$ admite que a veces sí que se comunica con diferentes personas para ampliar conocimientos.

Por último, y dada la escasa influencia, al menos de momento, de las redes sociales en el ámbito académico se preguntó al grueso del alumnado si cree que esta herramienta puede convertirse en un útil elemento de apoyo a la educación. En la mayor parte de los casos (71\%), la respuesta fue negativa.

\section{CONCLUSIONES}

El análisis del uso de las redes sociales entre jóvenes hispanohablantes ha dejado claro el uso socializador de las mismas, puesto que, como ya se ha destacado, la mayor parte de los estudiantes hacen uso de esta herramienta digital y pasan mucho tiempo conectados a Internet para comunicarse con las personas de su entorno. Esta es una premisa que podemos extrapolar a todos los discentes encuestados, ya que, independientemente de su nacionalidad, los datos (aunque varían ligeramente) son muy similares. Bernete (2009: 111) da cuenta de la importancia que han adquirido las redes sociales en la vida de los estudiantes: 
"Los jóvenes y adolescentes se unen a las redes porque es allí donde están sus amigos, además de estar en el Instituto de Secundaria y Bachillerato, en la calle y otros lugares públicos. Los entornos que suelen llamarse virtuales, donde se produce (se reproduce, se prolonga) el encuentro con los amigos y colegas están convirtiéndose en algo esencial para ser una persona integrada en aquellos círculos que cada uno tiene por grupos de referencia (a los que tiene que pertenecer)"

Estar en una comunidad virtual es vital para un estudiante. Sin embargo, hay un gran volumen de discentes de todas las nacionalidades que manifiesta no confiar en las mismas, por miedo a que se vulnere su intimidad, eso no es un impedimento para el empleo casi masivo, en muchos casos, de las redes sociales. De hecho, el estudio sobre redes sociales e Internet desarrollado por la empresa «Interactive Advertising Bureau» (2009) desvela que, pese a que la mayor parte de la población española posee Internet en casa y hace un uso casi diario de las redes sociales, el $37 \%$ manifiesta que hay cierta carencia de privacidad en esta herramienta virtual.

Además, también hemos podido comprobar que lo habitual es que los jóvenes dediquen entre dos y cinco horas diarias a la conexión a las redes sociales. Por países, los estudiantes mejicanos son los que irían a la cabeza, seguidos de los chilenos, los argentinos y los españoles. Ello también queda reflejado en el estudio de la empresa especializada en mediciones de Internet «comScore» (2011), que reveló que Argentina es el tercer país en el mundo cuyos usuarios pasan un mayor volumen de tiempo conectados a redes sociales, de tal manera que se llega a alcanzar una media de 8,4 horas al mes por persona. Otro país latinoamericano en este informe es Méjico, en el que se alcanzan las 5,3 horas mensuales.

Dicho lo anterior, podemos decir que resultaría lógico poder abordar ese tiempo de conexión mediante actividades académicas a través de la Red. Las opciones podrían ser, entre otras, crear páginas exclusivas para debatir un tema, propuestas de nuevas actividades a través de las redes sociales o creación de actividades «online» enlazadas a través de una comunidad virtual. Son muchas las iniciativas que se pueden llevar a cabo; los grupos de trabajo colaborativo son una opción que ya se está manejando en algunas materias y en diferentes niveles educativos, ya sea en estudios medios 0 superiores. Pueden generar actividades muy dinámicas, ya que la base de los mismos se encuentra en la interacción continua y permanente (Arroyo, Castro \& Peley, 2008: 121). En este sentido, Stojanovic de Casas (2006: 137) también matiza:

"Si se acepta que el aprendizaje online estimula el aprendizaje colaborativo, habrá que reconsiderar la forma de diseño y administración de un programa, lo cual requiere un enfoque distinto al de las primeras generaciones"

Además, y para reforzar lo anterior, también hemos de tener en cuenta que el lugar de procedencia del estudiante no le condiciona para hacer uso o no las redes sociales, puesto que hay una amplia mayoría que hace uso de las mismas, sobre todo si hablamos de estudiantes mayores de 18 años. Sin embargo, si hay que matizar que el país de residencia influye en el uso de una u otra red social; así por ejemplo, si 
hablamos de discentes chilenos, la tendencia es utilizar Facebook, mientras que si hablamos de españoles el protagonista es Tuenti.

Por tanto, el planteamiento de actividades académicas a través de las redes sociales debe pasar por el conocimiento previo de su uso por parte del alumnado. Además, el factor edad también se debe tener en cuenta en diferentes contextos, porque, si atendemos, por ejemplo, al caso de Argentina, los estudiantes de entre 12 y 18 años no están en ninguna comunidad virtual, mientras que el colectivo que supera los 18 sí que se encuentra en Facebook. Es decir, no con todos los grupos de discentes funcionaría la misma metodología didáctica a través de las redes sociales. De este modo, también debemos añadir que, para poder abordar el currículum académico a través de las redes sociales, hay que tener en cuenta el nivel del que partimos, ya que, como se ha comprobado, los estudiantes que hacen un mayor uso de esta herramienta son los que se encuentran en estudios superiores.

La edad como determinante del uso de las redes sociales también ha sido puesto de manifiesto por el Observatorio de Redes Sociales 3 , ya que, según el informe, Facebook es más utilizada por la población española con edades comprendidas entre los 26 y los 35 años. Tuenti, por su parte, y como también se ha señalado en este estudio, alcanza a un público más joven, que normalmente oscila entre los 16 y 25 años. De hecho, en nuestro estudio hemos podido comprobar que casi el $80 \%$ de los estudiantes de entre 16 y 18 hace uso de esta red social.

También es importante recordar que el alumnado suele pertenecer a una comunidad virtual para poder estar en contacto permanente con sus amigos/-as. De hecho, el estudio de Pérez-Díaz y Rodríguez (2008) pone de manifiesto también este aspecto al revelar que muchos jóvenes utilizan las redes sociales para sentir que siempre están en contacto con las personas de su entorno. Ello también lo confirma el estudio desarrollado por la empresa Interactive Adversing Bureau (2009), que añade que existe un claro predominio del uso de las redes sociales para estar en contacto con las amistades actuales más que con las del pasado.

Dicho todo lo anterior, y a tenor de los resultados obtenidos, podemos decir que, dado el alcance y relevancia actual que tienen las redes sociales en la vida de los jóvenes, sería factible unir su vida académica a su cotidinianeidad diaria, ahora en Internet. Por ello, y aunque la mayoría afirma que el uso de las redes sociales afecta negativamente a su vida académica, éstas podrían ser un elemento útil para dinamizar la enseñanza y para positivizar el tiempo de conexión (que a veces se traduce en pérdida de tiempo) del alumnado.

Del mismo modo que la educación virtual o «e-learning» ya es una realidad, tanto en estudios medios como superiores, una formación presencial complementada con el uso de las redes sociales podría generar nuevos y numerosos beneficios al colectivo de estudiantes.

Los espacios virtuales como soportes educativos generan una formación democrática basada en la optimización de la relación entre estudiantes, entre el colectivo discente 
y el docente, así como de cualquier otro miembro de la comunidad con la organización y viceversa (Sangrá, 2001:122).

$\mathrm{F}$

inalmente, y una vez expuestos todos los criterios que han dado fruto a esta investigación, hemos de señalar que se debería seguir ahondando en esta línea. No debemos olvidar que las redes sociales protagonizan las rutinas diarias de todos los estudiantes, y la continua investigación en esta línea facilitaría una mayor y mejor relación entre la comunidad docente y el alumnado.

\section{BIBLIOGRAFÍA}

Arroyo, E; Castro, E.; Rosario, P. (2008): "La educación y la web semántica". Télématique: Revista Electrónica de Estudios Telemáticos 7: 117-126. Maracaibo: Universidad Rafael Belloso Chacón.

Aznar, V; Soto, J. (2010): "Análisis de las aportaciones de los blogs educativos al logro de la competencia digital". Revista de Investigación en Educación 7: 83-90. Vigo: Facultad de Ciencias de la Educación y el Deporte, Universidad de Vigo.

Barron, A.; Gary W.O. (1997): New technologies for education: a beginner's guide. Colorado: Librairies Unlimited.

Barros, B.; Read, T.; Verdejo, M. F. (2008): Virtual Collaborative Experimentation: an Aproach Combining Remote and Local Labs. IEEE Education Society: Iowa

Bernete, F. (2010): "Usos de las TIC. Relaciones sociales y cambios en la socialización de las y los jóvenes". Revista de Estudios de Juventud 88: 97-114. Madrid: INJUVE.

Castells, M. (2001): La Galaxia Internet. Barcelona: Plaza \&Janés Editores.

Celaya, J. (2008): La empresa en la Web 2.0. Barcelona: Gestión 2000.

Langer Georgea, M. (2003): Collaborative analysis of student work: improving teaching and learning. Alexandria: Association for Supervision and Curriculum Development.

FUNDACIÓN TELEFÓNICA (2010): La Sociedad de la Información en España 2010. Madrid: Editorial Ariel.

Ghiglione, R.; Matalon, B. (1989): Las encuestas sociológicas. Méjico: Trillas

Pérez-Díaz, V.; Rodríguez, J.C. (2008): La adolescencia, sus vulnerabilidades y las nuevas tecnologías de la información y la comunicación. Madrid: Fundación Vodafone España. 
Parra Castrillón, E. (2010): "Las redes sociales de Internet: también dentro de los hábitos de los estudiantes universitarios". Tendencias. Revista de la Facultad de Ciencias Económicas y Administrativas 2: 193-207. Colombia: Universidad de Nariño.

Sangrá, A. (2001): "Enseñar y aprender en la virtualidad". Educar 28: 117-131. Barcelona: Universidad Autónoma de Barcelona.

Stojanovic de Casas, L. (2006): "Las tecnologías de la información y comunicación en la promoción de nuevas formas interactivas y de aprendizaje en la Educación a Distancia". Revista de investigación 59: 121-140. Caracas: Instituto Pedagógico de Caracas.

Visauta, B. (1989): Técnicas de Investigación social. I, Recogida de datos. Barcelona: PPU

Weert, T. (2005): Information and Communication Technologies and Real-Life. Boston: International Federation for Information Processing.

\section{María del Mar Soria Ibáñez}

Periodista en activo. Trayectoria en prensa, televisión y radio. Doctora en Publicidad y Relaciones Públicas, especializada en la Comunicación en el Tercer Sector. Actualmente profesora del CES Felipe II, adscrito a la Universidad Complutense de Madrid. Profesora participante en programas de posgrado y de especialización de la Universidad de Málaga 